Paper Title:

\title{
A Comparison of Logistic and Harvey Models for Electricity Consumption in New Zealand
}

Authors : $\quad$ Zaid Mohamed ${ }^{1}$ and Pat Bodger ${ }^{*}$,

Affiliations:

1. Mohamed, Z., B.E (Hons), is a Ph.D. student in the Department of Electrical and Computer Engineering at the University of Canterbury, Christchurch, New Zealand.

e-mail: zmo10@student.canterbury.ac.nz

2. Bodger, P.S, B.E (Hons), Ph.D., is Professor of Electric Power Engineering, Department of Electrical and Computer Engineering University of Canterbury, P.O Box 4800, Christchurch, New Zealand, Phone: (64)3642070, Fax: (64)3642761 e-mail: p.bodger@elec.canterbury.ac.nz

Running title: Harvey Model for New Zealand Electricity

${ }^{*}$ Corresponding author 


\begin{abstract}
There have been a number of forecasting models based on various forms of the logistic growth curve. This paper investigates the effectiveness of two forms of Harvey models and a Logistic model for forecasting electricity consumption in New Zealand. The three growth curve models are applied to the Domestic and Non-Domestic sectors and Total electricity consumption in New Zealand. The developed models are compared using their goodness of fit to historical data and forecasting accuracy over a period of 19 years. The comparison revealed that the Harvey model is a very appropriate candidate for forecasting electricity consumption in New Zealand. The developed models are also compared with some available national forecasts.
\end{abstract}

Keywords: Forecasting; Energy; Growth curve models; Logistic growth 


\section{Introduction}

Logistic models are attractive in situations where there is thought to be a saturation level to a time series. A number of researchers have investigated the Logistic model either in its simplest form or in a modified form, in studying various technological changes [1-10]. The Logistic model has also been successful in forecasting electricity consumption [11$16]$.

The Logistic model $[11,12]$ uses a Fibonacci search technique to determine the saturation level. In that model, the saturation level needs to be estimated before the required parameters of the logistic model may be estimated. It was found that the Logistic model was very effective in describing the historical electricity consumption in New Zealand but produced forecasts lower than the available national forecasts supporting the perception that the logistic bias underestimates the final ceiling. This is mainly due to the constraints imposed by the saturation level of the logistic growth curve. However, underestimating the final ceiling is not always a characteristic of the logistic growth model as applying it to the early growth data may lead to higher values. A time series forecasting model based on the logistic curve was proposed by Harvey $[17,18]$. The Harvey models do not require a saturation level to be estimated prior to estimation of the parameters. However, the model approaches a saturation level with time. There are two forms of Harvey models; a Harvey Logistic Model based on the general logistic model and a Harvey Model based on general modified exponentials [17]. In general, the logistic growth is growth in competition, while exponential growth represents a "population' 
explosion typically encountered during the early phases of logistic growth. The Harvey model constitutes an admixture of logistic and exponential growth. This paper investigates the effectiveness of two forms of the Harvey models [17] for electricity consumption in New Zealand and compares them with the previously developed Logistic model $[11,12]$.

\section{Model Theory}

\subsection{Logistic and Harvey Logistic Model}

Univariate time series models are often based on a local, rather than a global trend [17]. In local trend models, recent observations receive more weight when forecasting than those in the more distant past. In global trend models, the time path of the data concerned is regarded as following a deterministic function of time, upon which a disturbance or error term is added.

Electricity consumption, $f(t)$, can be represented by the Logistic function as,

$$
f(t)=\frac{\alpha}{1+\beta e^{\gamma}} \quad 1 \leq t \leq T
$$


where, $\alpha$ is the saturation level

$\beta$ and $\gamma$ are parameters to be estimated

$t$ is the time in years

In the Logistic model, $\alpha$ is estimated by a Fibonacci search technique [11, 12].

When Eq. (1) is differentiated with respect to $t$ and natural logarithms taken on both sides, the following equation is obtained [17],

$$
\ln \frac{d f(t)}{d t}=2 \ln f(t)+\delta+\gamma t
$$

where,

$$
\delta=\ln \left(\frac{-\beta \gamma}{\alpha}\right)
$$

Using Eq. (2), the proposed Harvey Logistic Model is [17],

$$
\ln y_{t}=2 \ln Y_{t-1}+\delta+\gamma t+\varepsilon_{t}, \quad t=2 \ldots T
$$

where,

$$
\begin{aligned}
& Y_{t} \text { is the electricity consumption at year } t . \\
& y_{t}=Y_{t}-Y_{t-1}, \quad t=2 \ldots T
\end{aligned}
$$


$\varepsilon_{t}$ is a disturbance term with zero mean and constant variance

$\delta$ and $\gamma$ are constants to be found by regression.

Eq. (3) is rearranged to give:

$$
\ln \left(\frac{y_{t}}{Y_{t-1}^{2}}\right)=\delta+\gamma+\varepsilon_{t}
$$

The parameters $\delta$ and $\gamma$ are found by regressing $\ln \left(\frac{y_{t}}{Y_{t-1}^{2}}\right)$ on $t$. Eq. (4) can be written as,

$$
y_{t}=Y_{t-1}^{2} e^{(\delta+\gamma)}
$$

It can be seen that Eq. (5) no longer contain the error term, $\varepsilon_{t}$. This is to simplify the

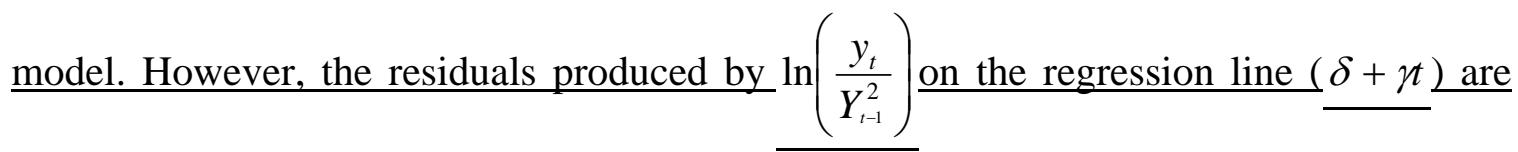
studied using Durbin-Watson (DW) $d$-statistics [19]. Upon acceptable DW statistics, the models are fitted to the data sets. More details can be found in Section 3.

Since $y_{t}=Y_{t}-Y_{t-1}$, then Eq. (5) can be written as,

$$
Y_{t}=Y_{t-1}+Y_{t-1}^{2} e^{(\delta+\gamma)}
$$

The $h$-step ahead forecasts of the electricity consumption, $\hat{Y}$, can be made by using, 


$$
\hat{Y}_{t+h}=\hat{Y}_{t+h-1}+\hat{Y}_{t+h-1}^{2} e^{(\delta+\gamma(t+h))}
$$

The forecast for electricity consumption takes the form of the Logistic curve and gradually approaches the saturation level $\alpha$.

\subsection{Harvey Model}

The general modified exponential function is of the form [17],

$$
f(t)=\alpha\left(1+\beta e^{\gamma t}\right)^{k}
$$

The value of $k$ determines the form of the function $f(t)$. When $k=-1, f(t)$ is Logistic and when $k=1$ it is a simple modified exponential.

Differentiating and the taking natural logarithm as for the Logistic model, leads to the Harvey model [17]:

$$
\ln y_{t}=\rho \ln Y_{t-1}+\delta+\gamma+\varepsilon_{t}
$$

where,

$$
\begin{aligned}
& \rho=\frac{k-1}{k} \\
& \delta=\ln \left(k \beta \alpha^{1 / k} \gamma\right)
\end{aligned}
$$


$\rho, \beta$ and $\gamma$ are parameters to be estimated.

Forecasts are obtained using:

$$
\hat{Y}_{t+h}=\hat{Y}_{t+h-1}+\hat{Y}_{t+h-1}^{\rho} e^{(\delta+\gamma(t+h))}
$$

Mean absolute percentage error (MAPE) and the Durbin-Watson statistic (DW) are used in the comparison of the models [19]. MAPE gives an indication of the goodness of the fit of the model to the historical data. MAPE is also used to compare the forecasting accuracy of the models. The DW statistic tests whether the residuals of the fitted model are independent. A DW statistic close to 2 indicates that there is no correlation in the errors produced by the developed model.

\section{Application to New Zealand Electricity Consumption}

The annual electricity consumption data for New Zealand [20,21] from 1943 to 1999 are modeled using the Harvey Logistic and Harvey Model. The models are applied separately to each of the Domestic and the Non-Domestic sectors and to the Total consumption data. Domestic and Non-domestic sectors are often studied separately because of their perceived difference in contribution to society. The Domestic sector of residential customers is primarily a goods and services consumption sector of society while the Non- 
Domestic sector is the production of goods and services and hence that which gives rise to the generation of economic wealth of a country. It never the less consumes electricity (and other resources) in generating that wealth. The Total consumption is simply the total electricity consumed and is the aggregate of the Domestic and the Non-Domestic sector consumptions. In addition these are the sectors that the government has used for electricity forecasting and are well accepted and published data sets. Regressing $\ln \left(\frac{y_{t}}{Y_{t-1}^{2}}\right)$ over the time period 1943-1999 gives the following Harvey Logistic models.

$$
\begin{array}{ll}
\text { Domestic: } & \ln y_{t}=2 \ln Y_{t-1}+150.86-0.083 t \\
\text { Non-Domestic: } & \ln y_{t}=2 \ln Y_{t-1}+145.79-0.080 t \\
\text { Total: } & \ln y_{t}=2 \ln Y_{t-1}+145.60-0.081 t
\end{array}
$$

A plot of $\ln \left(\frac{y_{t}}{Y_{t-1}^{2}}\right)$ along with the fitted regression line for the Domestic sector is shown in Fig. 1.

The residuals are very well behaved with a Durbin-Watson (DW) statistic of 2.0. The residuals are also reasonably well behaved in the Non-Domestic sector and Total consumption, with Durbin-Watson statistics of 1.1 and 1.5, although there is some indication of serial correlation in the case of Non-Domestic data. Fig. 2 shows the fitted Harvey Logistic models for the historical electricity consumption. 
The Harvey Logistic models have produced very good fits of the historical electricity consumption with MAPE values of 3.1 for Domestic, 3.3 for Non-Domestic and 2.6 for Total consumption data.

Application of the electricity consumption data to the Harvey Model (Eq. 9) resulted in the following models.

$$
\begin{array}{ll}
\text { Domestic: } & \ln y_{t}=0.60 \ln Y_{t-1}+35.44-0.018 t \\
\text { Non-Domestic: } & \ln y_{t}=1.29 \ln Y_{t-1}+57.46-0.032 t \\
\text { Total: } & \ln y_{t}=1.08 \ln Y_{t-1}+50.27-0.028 t
\end{array}
$$

Where, $t$ is the time in years from 1944 to 1999.

These Harvey models also produced very good fits with MAPE values of 3.1 for Domestic, 3.3 for Non-Domestic and 2.7 for Total consumption. These values are very close to the Harvey Logistic model fits and thus the fitted Harvey models for the historic periods of the Domestic and Non-Domestic sectors and Total consumption are very similar to those shown in Fig. 2. However, the coefficients of $Y_{t-1}$ (Eqs. 14-16) are significantly different from the 2 of those in the Harvey Logistic models (Eqs. 11-13). These values indicate that the Harvey models are different from the Harvey Logistic models. 


\section{Comparison of Forecasts}

\subsection{Comparison of the Harvey and Logistic Models}

\subsubsection{Goodness of Fit and Future Consumptions}

Forecasts produced by the Harvey models together with the forecasts of the Logistic models for the Domestic, Non-Domestic and Total consumption are shown in Fig. 3, Fig. 4 and Fig. 5 respectively.

The forecasts for the Domestic and Non-Domestic sectors and Total consumptions are increasing in an exponential nature for the three models. The Harvey model has given rise to the highest forecasts for the three data sets considered. The Logistic model forecasted the lowest consumptions for the three data sets. The Harvey Logistic model gave forecasts somewhere in between the other two forecasts.

The MAPE and DW values of the fitted models from 1943 to 1999 for the Logistic, Harvey Logistic and Harvey models are given in Table 1.

The MAPE values are very similar for the Harvey Logistic and Harvey models. In addition, the lowest MAPE values are also recorded for these models. This indicates that the Harvey models provide better fits of the historical data than the Logistic models. In addition, the Durbin-Watson statistics are closer to 2 in the Harvey Logistic and Harvey models. This indicates that the residuals are more reasonably well behaved in the Harvey 
Logistic and Harvey models compared to the Logistic models. The DW values are much smaller than 2 in the Logistic models indicating that there are some positive autocorrelation in the residuals.

The resulting low predictions by the Logistic models are due to the constraints imposed by the saturation level. In proposing the Logistic model [11,12] the saturation level is obtained by the Fibonacci search technique prior to obtaining the constants by regression analysis. In the Harvey Logistic model the asymptote is not approximated prior to the regression analysis. As a result, the curve of the Harvey Logistic model gradually approaches a saturation level. This has given rise to higher forecasts than the Logistic model. The Harvey model is different from the other two in the sense that it has got one extra parameter, $\rho$, to be estimated as a part of the regression analysis. It has been shown that the parameter, $\rho$, calculated for each of the Domestic and Non-Domestic sectors and Total consumption are significantly different from 2 showing that for these data, the Harvey model is not equivalent to the Harvey Logistic model. However, with as good fits to historical data as the Harvey Logistic model, the Harvey model has given rise to higher consumption forecasts overall.

\subsubsection{Forecasting Accuracy}

The Logistic, Harvey Logistic and Harvey models are further analyzed for forecasting accuracy. A number of actual consumption data points at the end of the series are held out for comparison with the forecasts obtained by the developed models. The forecasts 
for the 9 years ahead and 19 years ahead, when the last 9 years and 19 years of data were held out for the Total electricity consumption are shown in Fig. 6.

For the 9 years ahead forecasts the Harvey model gave forecasts that are closest to the actual values while in the 19 years ahead forecasts, the Logistic model gave forecasts that are very close to the actual Total consumption values. This suggests the choice of the best model should not be made by just looking at the forecasts of the two chosen periods. Therefore, forecasting models are obtained with data values held out from 1 year through to 19 years for each of the three models. The average MAPE values of each of the models using the actual values held out for each of the forecasted period from 1 year through to 19 years are shown in Fig. 7, for the Domestic and Non-Domestic sectors and Total consumption respectively.

For the Domestic sector, the Harvey model has given the lowest MAPE values from 1year through to 18 -years ahead. The Harvey Logistic model gave very similar values to the Harvey model with slightly larger errors. The Logistic model gave the highest error values except at the 19-years ahead forecast. This indicates that the Harvey model is the best among these three in forecasting the Domestic consumption for a period of up to 18 years ahead.

For the Non-Domestic sector, it is the Logistic model that gave the overall MAPE values from 1-year ahead through to 18-years ahead forecasts. However, the Harvey Logistic gave the lowest errors in the initial 6 years. The Harvey model also gave acceptable 
results in the initial 8 years. Overall, the Logistic model is the best model for forecasting electricity consumption in the Non-Domestic sector, especially for longer horizons up to 18 years ahead.

For the Total consumption, the errors are more comparable. However, the Harvey model gave the lowest errors from 1-year ahead through to 14-years ahead forecasts while the Logistic model performed better from 15-years through to 19-years ahead forecasts. The Harvey Logistic model gave similar results to the Harvey model, but the errors are slightly larger than for the Harvey model. These results indicate that the Harvey model is the best to forecast Total electricity consumption for periods from 1 year through to 15 years ahead.

Young [15] studied nine different growth curve models including the Logistic and Harvey models by comparing MAPE. The comparison revealed that the Harvey model was one of the three proposed models for forecasting time series with an unknown upper limit. The analysis in this section supports those results, indicating that the Harvey model is an appropriate forecasting model for New Zealand electricity consumption.

A good forecasting model is often selected on the ability of the model to describe the future data and not necessarily gives the best fit of the historical data [22]. The Harvey model not only generated the best fit of the future data, but was also among the best in fitting historical data. This strengthens the choice of the Harvey model in forecasting electricity consumption in New Zealand. 


\subsection{Comparison with National Forecasts}

The forecasts obtained by the Logistic, Harvey and Harvey Logistic models are compared with the national forecasts available in New Zealand. They are CAE models [23] and MED models [24]. The MED forecasts are made by the Ministry of Economic Development, New Zealand, using its SADEM energy supply and demand model. The SADEM model is a descriptive market equilibrium model focusing on the entire energy sector. The model determines equilibrium in the energy market by projecting demands for a given set of prices and comparing this with the modelled cost of supplying this level of demand [24]. The CAE forecasts are modelled using an annual load growth of $1.8 \%$. Their study has used $1.8 \%$ as the baseline estimate, with $1.3 \%$ and $2.3 \%$ growth used for sensitivity analysis. This paper uses the $1.8 \%$ baseline estimate for comparison purposes.

The forecasts obtained by these models for the Domestic and Non-Domestic sectors and Total consumption are shown in Fig. 8, Fig. 9 and Fig. 10 respectively.

For the Domestic sector, the Logistic model forecasted the lowest consumption. The Harvey model forecasts are also lower than the other models, but somewhere in between the CAE and Harvey Logistic model forecasts. For the Non-Domestic sector, the forecasts of the Harvey Logistic model are very close to the CAE and MED model forecasts while the Harvey model forecasted the highest and the Logistic model forecasted the lowest consumption. 
For the Total consumption, the Harvey model forecasted very similar forecasts to the CAE and MED forecasts. The forecasts of the Logistic and Harvey Logistic models are much lower. The forecasts by the Harvey model are virtually indistinguishable from the CAE model and MED model forecasts. The fit to the historical data and forecasting accuracy indicates that the Harvey model is an excellent candidate in forecasting New Zealand electricity consumption.

\section{Summary}

This paper has investigated two forms of the Harvey models and compared them with a previously developed Logistic model for forecasting electricity consumption in New Zealand. It was found that the proposed models are generally appropriate in forecasting electricity consumption New Zealand. However, the proposed Harvey model has performed better than the Logistic model in most cases in terms of model fit to the historical data and forecasting accuracy. The Harvey model forecasted higher consumption comparable with national forecasts, especially for the Total consumption for New Zealand. The good model fit and forecasting accuracy has indicated that the Harvey model is a very suitable candidate in forecasting New Zealand electricity consumption. 


\section{Acknowledgements}

The authors gratefully acknowledge the helpful suggestions of two anonymous reviewers that enabled to improve the paper.

\section{References:}

[1] Carrillo, M., and Gonzalez, J.M.: A New Approach to modeling sigmoid curves, Technological Forecasting and Social Change 69, 233-241 (2002).

[2] Bass, F.M.: A new product growth for model consumer durables, Management Science 15(5), 215- 227 (1969).

[3] Jain, D.C., and Rao, R.C.: New concepts and applications in growth phenomena, Journal of Applied Statistics 21(3), 161-190 (1994).

[4] Bhargava, S., Bhargava, R., and Jain, A.: Requirement of dimensional consistency in model equations: Diffusion models incorporating price and their applications, Technological Forecasting and Social Change 41, 177-188 (1991).

[5] Frank, L.D.: An analysis of the effect of the economic situation on modeling and forecasting the diffusion of wireless communications in Finland, Technological Forecasting and Social Change, ARTICLE IN PRESS, 1-13 (2003).

[6] Bewley, R., and Fiebig, D.G.: A flexible logistic growth model with applications in telecommunications, International Journal of Forecasting 4, 177-192 (1988). 
[7] Meyer, P.S., and Ausubel, J.H.: Carrying capacity: A model with logistically varying limits, Technological Forecasting and Social Change 61, 209-214 (1999).

[8] Giovanis, A.N., and Skiadas, C.H.: A stochastic logistic innovation diffusion model studying the electricity consumption in Greece and the United States, Technological Forecasting and Social Change 61, 235-246, (1999).

[9] Mar-Molineo.: Tractors in Spain: A logistic analysis, Journal of Operational Research Society 31, 141-152 (1980).

[10] Oliver, F.R.: Tractors in Spain: A further logistic analysis, Journal of the Operational Research Society 32, 499 - 502.

[11] Mohamed, Z., and Bodger, P.S.: Analysis of the Logistic model for Predicting New Zealand Electricity Consumption, Proceedings of the Electricity Engineer's Association (EEA) New Zealand 2003 Conference, Christchurch, New Zealand, Published in CD-ROM, 20-21 June (2003).

[12] Bodger, P.S., and Tay, H.S.: Logistic and Energy Substitution Models for Electricity Forecasting: A Comparison Using New Zealand Consumption Data, Technological Forecasting and Social Change 31, 27-48 (1987).

[13] Skiadas, C.H., Papayannakis L.L., and Mourelatos, A.G.: An Attempt to Improve Forecasting Ability of Growth Functions: The Greek Electric System, Technological Forecasting and Social Change 44, 391- 404 (1993).

[14] Sharp, J.A., and Price, H.R.: Experience Curve Models in the Electricity Supply Industry, International Journal of Forecasting 6, 531-540 (1990).

[15] Young, P.: Technological Growth Curves: A Competition of Forecasting Models, Technological Forecasting and Social Change 44, 375-389 (1993). 
[16] Tingyan, X.: A Combined Growth Model for Trend Forecasts, Technological Forecasting and Social Change 38, 175-186 (1990).

[17] Harvey, A.C.: Time series forecasting based on the logistic curve, Journal of the Operational Research Society 35(7), 641-646 (1984).

[18] Harvey, A.C., Time Series Models. 2nd ed., The MIT Press, Cambridge, Massachusetts, 1993, p.149-152.

[19] Makridakis, S., Weelwright S.C., and Hyndman R.J.: Forecasting: Methods and Applications. Third ed., John Wiley and Sons, Inc., New York, 1998.

[20] Ministry of Energy.: Electricity Forecasting and Planning: A Background Report to the 1984 Energy Plan, issues of 1982. -1984.

[21] Ministry of Economic Development.: New Zealand Energy Data File, July 2002.

[22] Martino, J.P.: A review of selected recent advances in technological forecasting, Technological Forecasting and Social Change 70, 719-733 (2003).

[23] Sinclair Knight Merz and CAE (Centre for Advanced Engineering, University of Canterbury, NZ).: Electricity Supply and Demand to 2015. Fifth Edition, April 2000.

[24] Ministry of Economic Development, Modelling and Statistics Unit.: New Zealand Energy Outlook to 2020, February 2000. 
Table 1

MAPE and DW values for the fitted models $(\mathrm{D}=$ Domestic and ND = Non-Domestic $)$

\begin{tabular}{lcccccc}
\hline \multirow{2}{*}{ Model } & \multicolumn{3}{c}{ MAPE } & \multicolumn{3}{c}{ DW } \\
\cline { 2 - 7 } & D & ND & Total & D & ND & Total \\
\hline Logistic & 4.4 & 8.6 & 4.1 & 0.35 & 0.57 & 0.50 \\
\hline Harvey Logistic & 3.1 & 3.3 & 2.6 & 1.96 & 1.56 & 1.71 \\
\hline Harvey & 3.1 & 3.3 & 2.7 & 1.89 & 1.55 & 1.67 \\
\hline
\end{tabular}




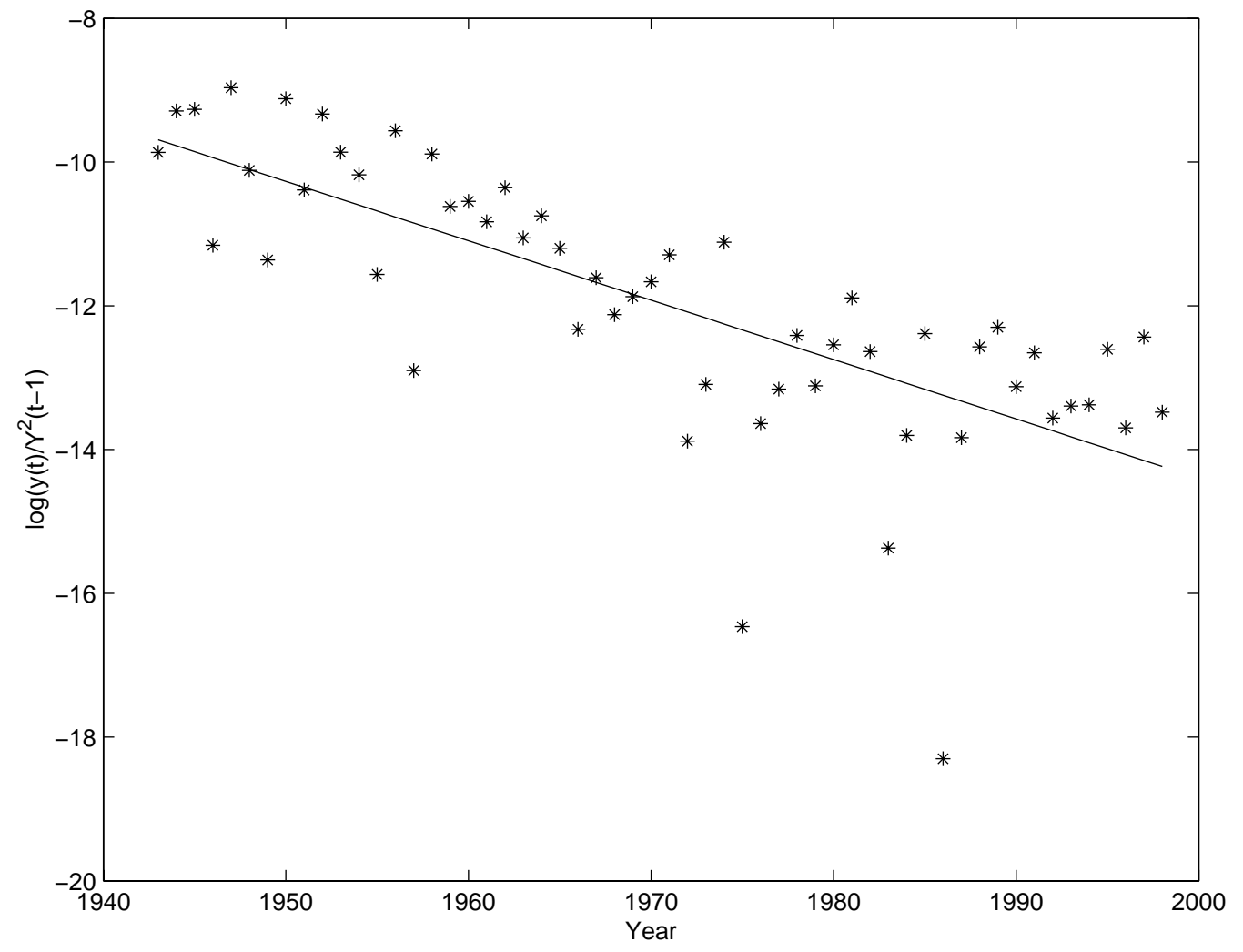

Fig.1. Plot of the regression line for Domestic sector 

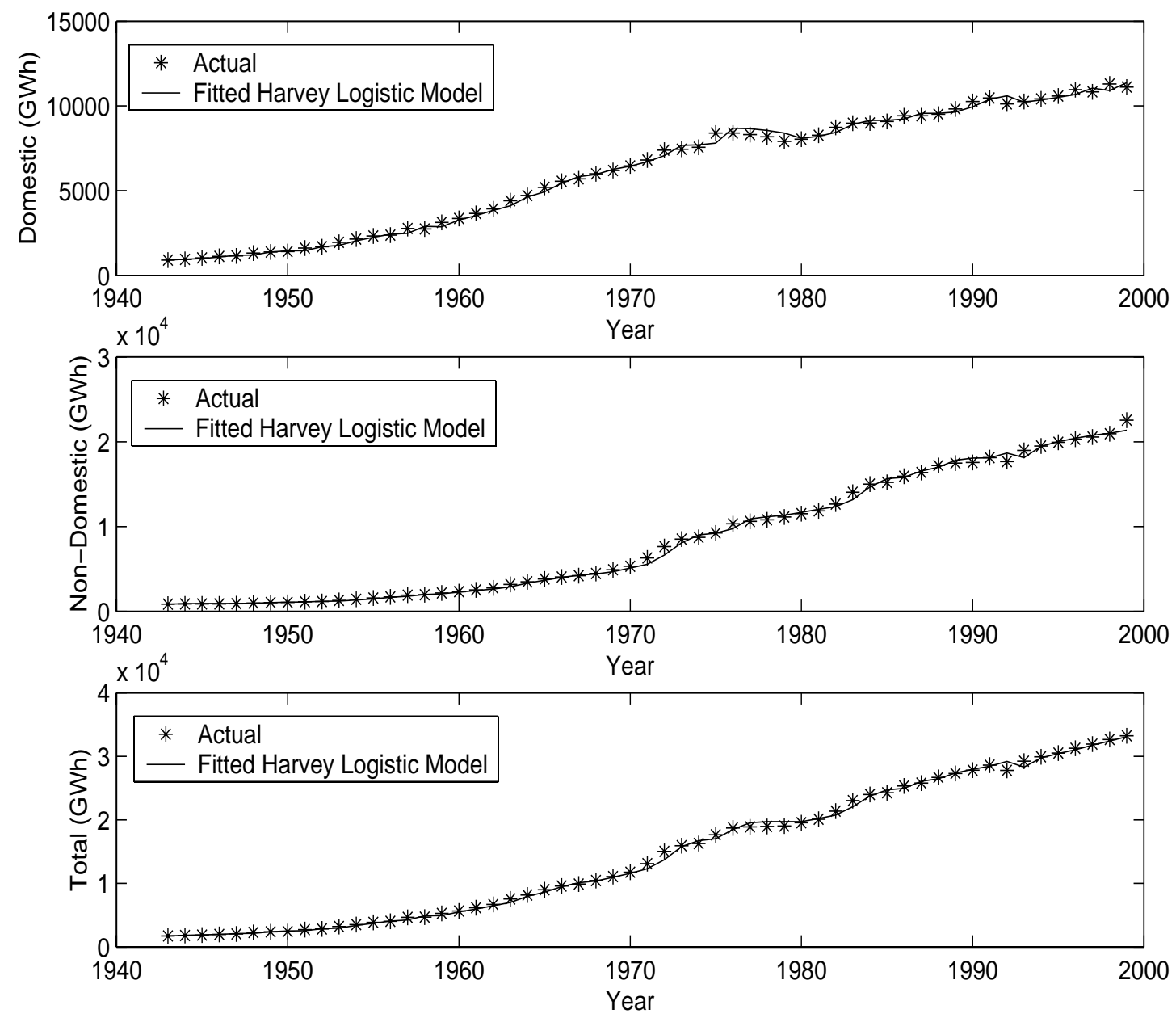

Fig. 2. Fitted Harvey Logistic models for the historical electricity consumption 


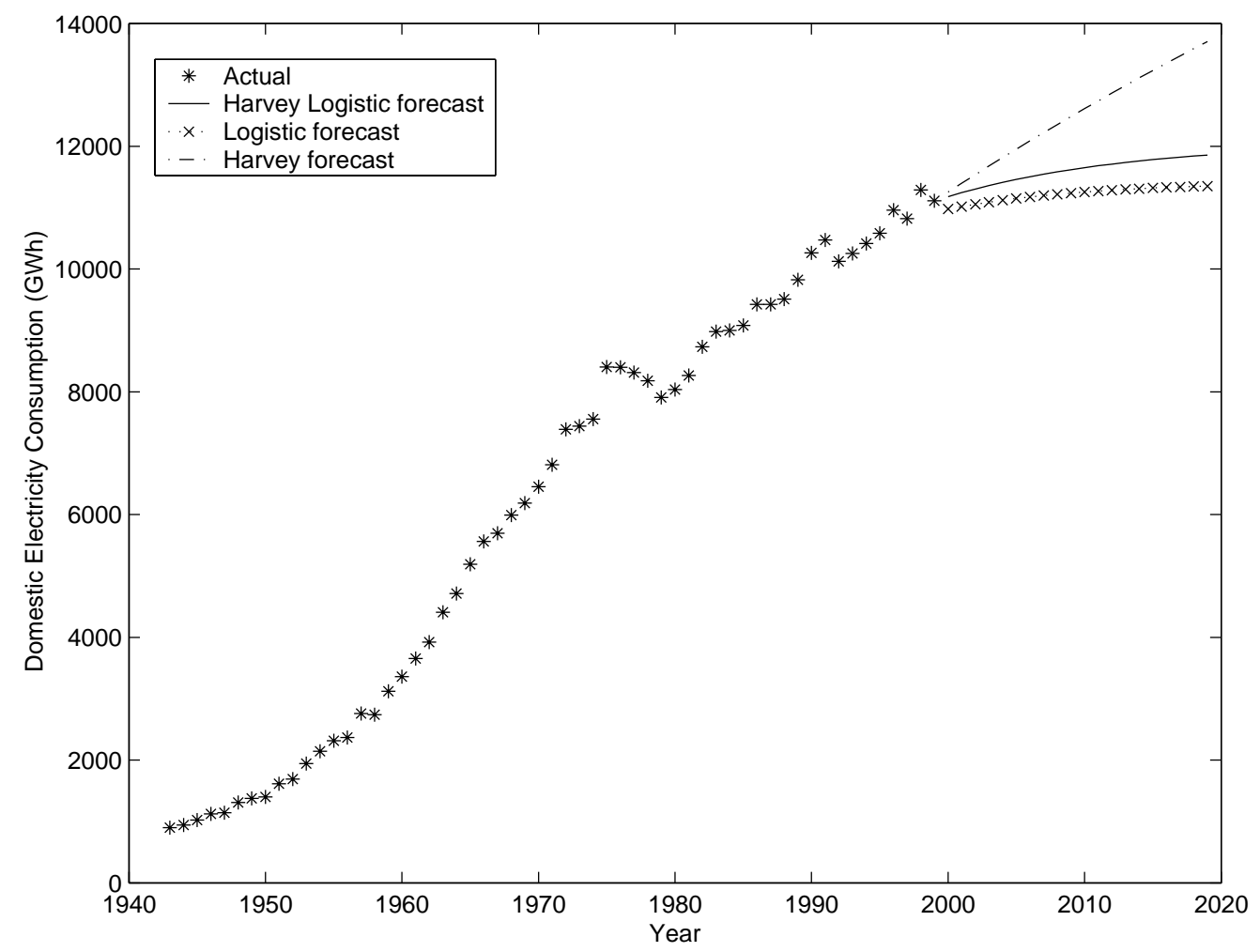

Fig. 3. Forecasts of the Logistic and Harvey models for Domestic electricity consumption 


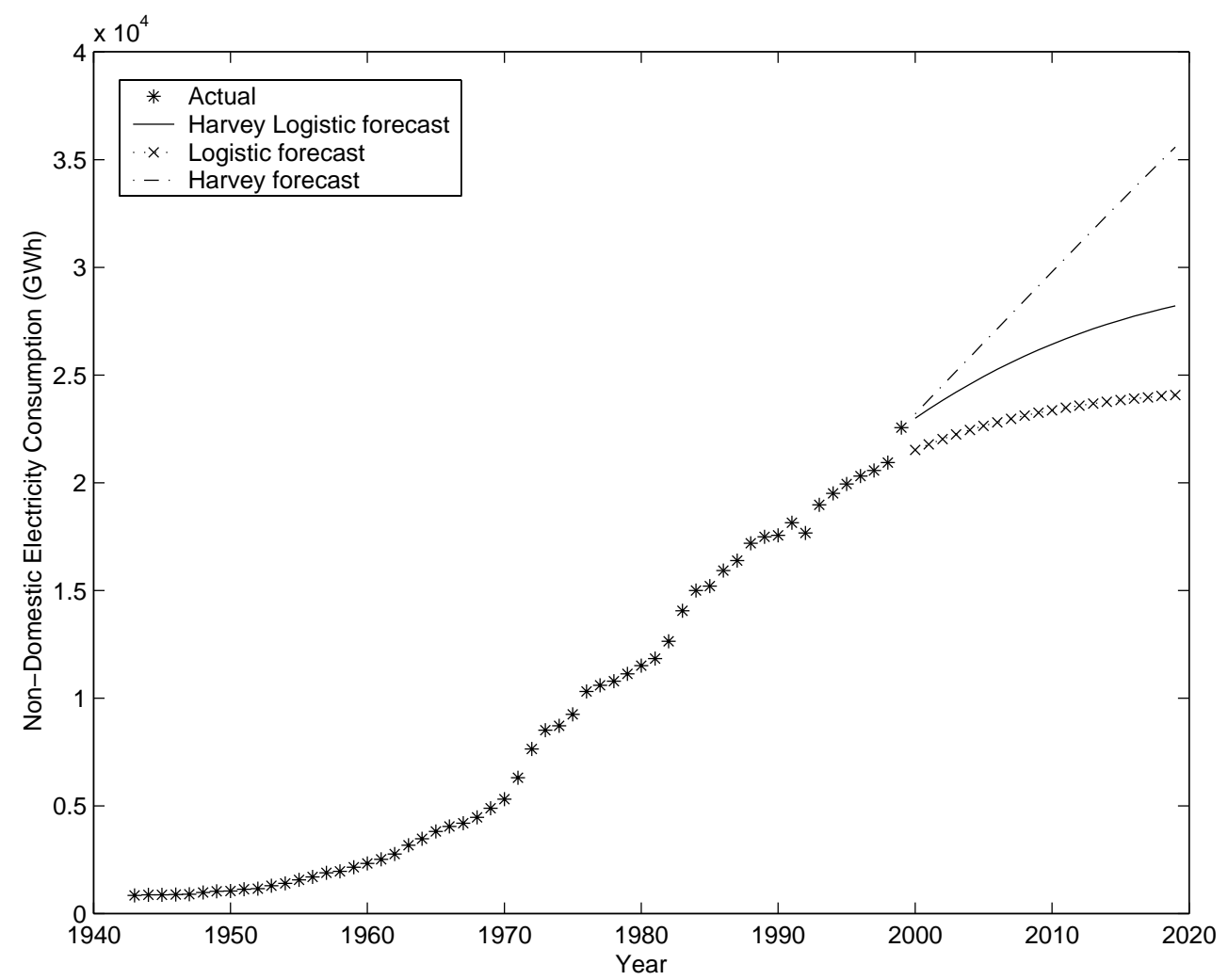

Fig. 4. Forecasts of the Logistic and Harvey models for Non-Domestic electricity consumption 


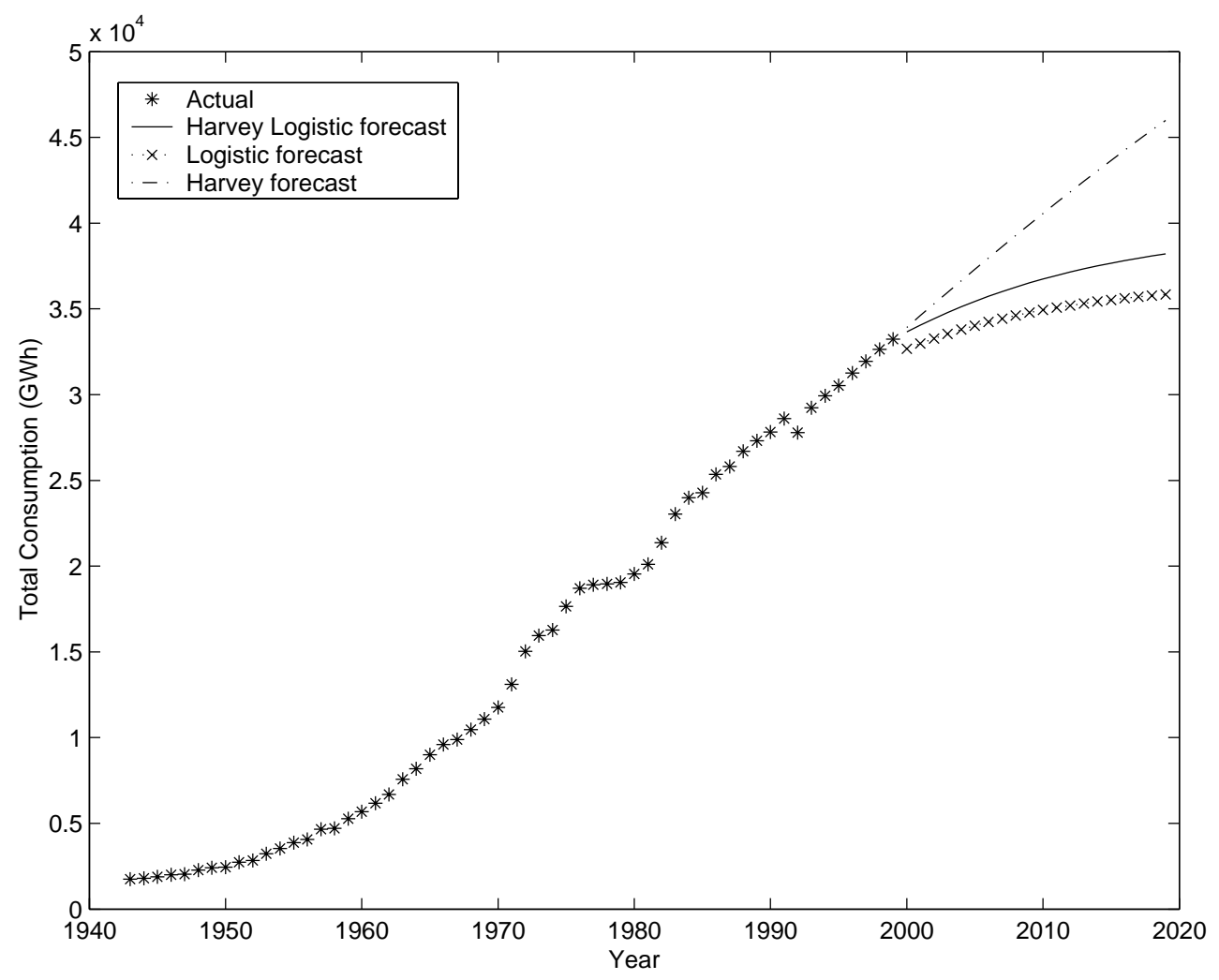

Fig. 5. Forecasts of the Logistic and Harvey models for Total electricity consumption 

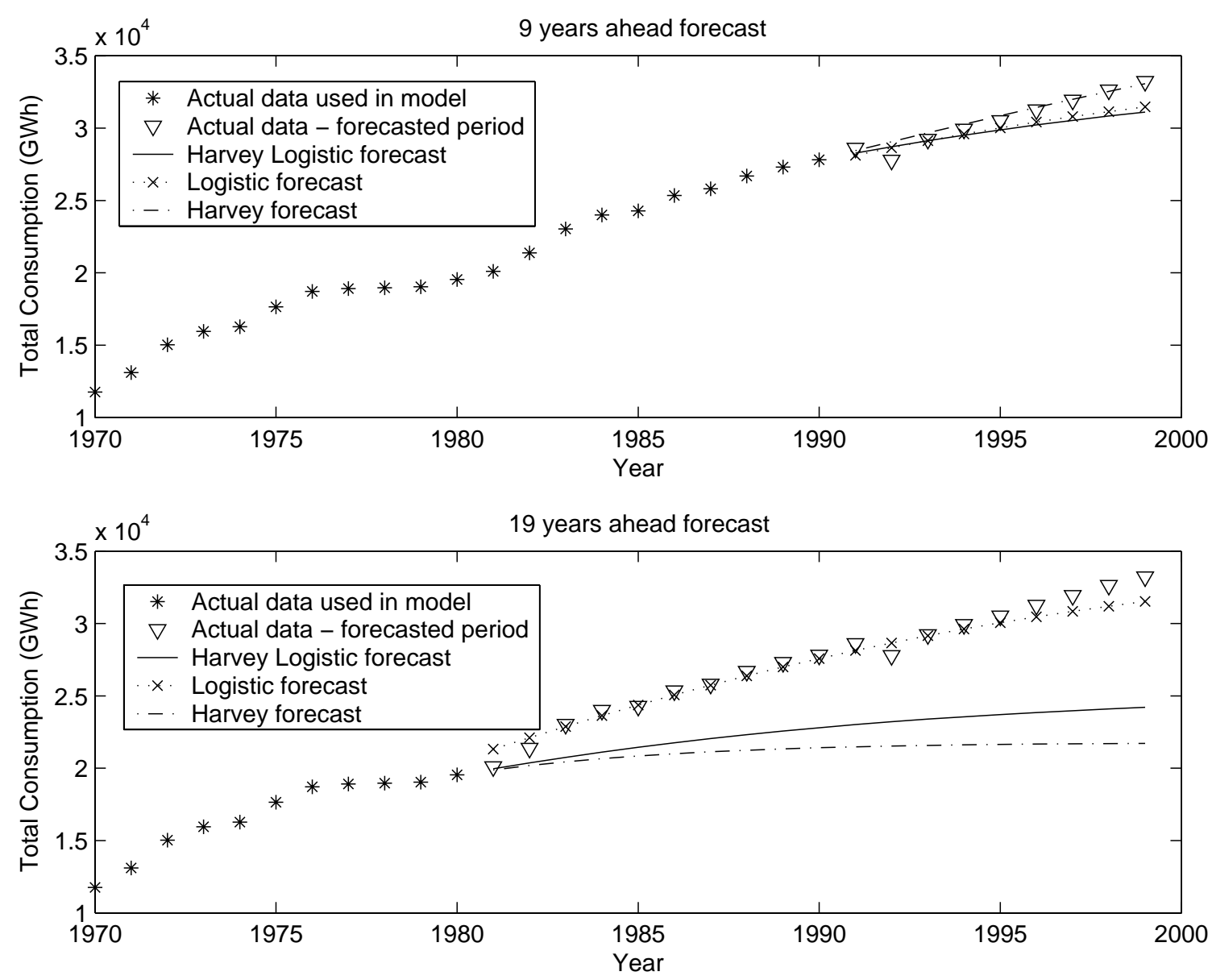

Fig. 6. Comparison of Total electricity consumption forecasts for 9 years and 19 years ahead 

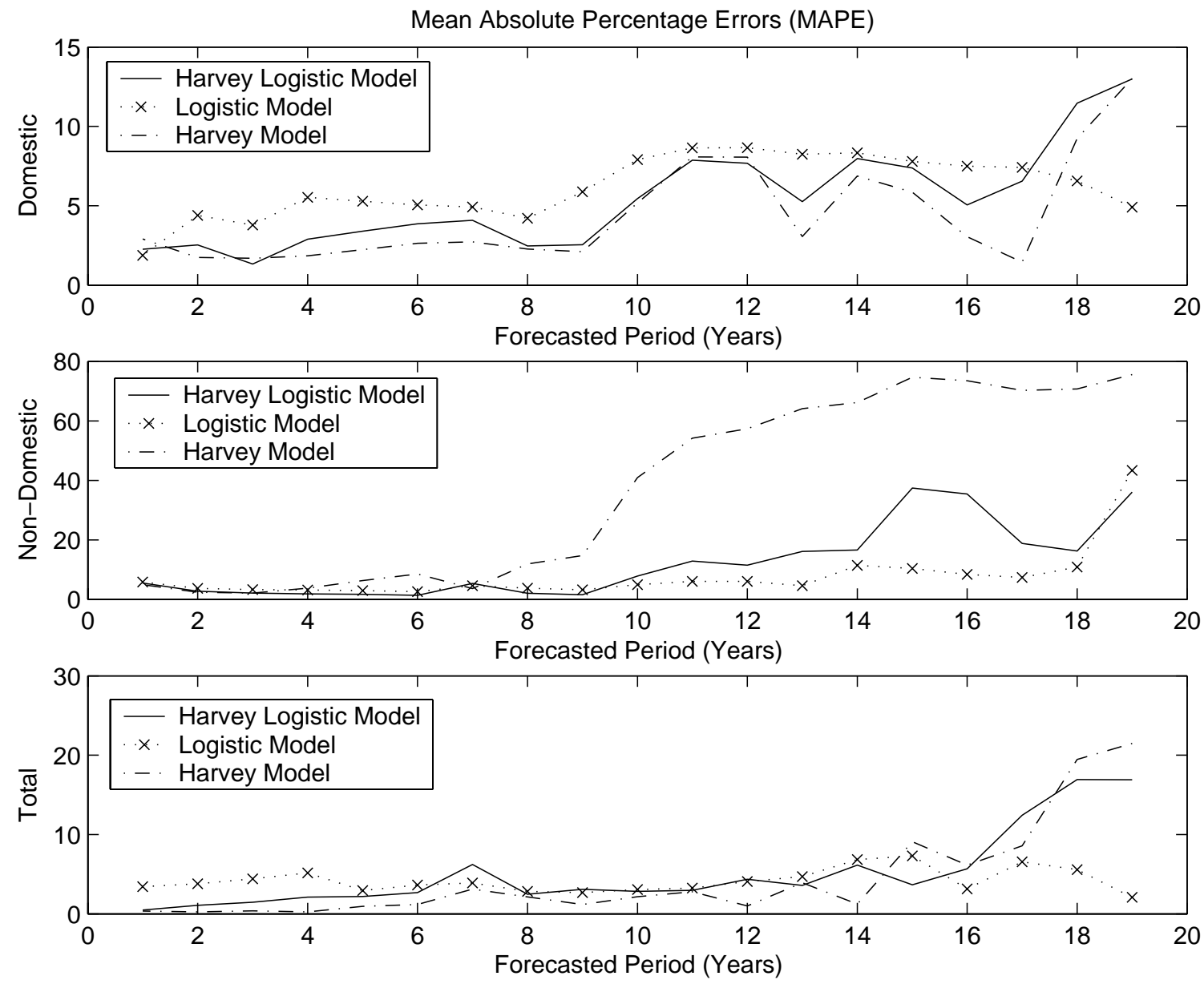

Fig. 7. Forecasting accuracy from 1 year to 19 years for the three models. 


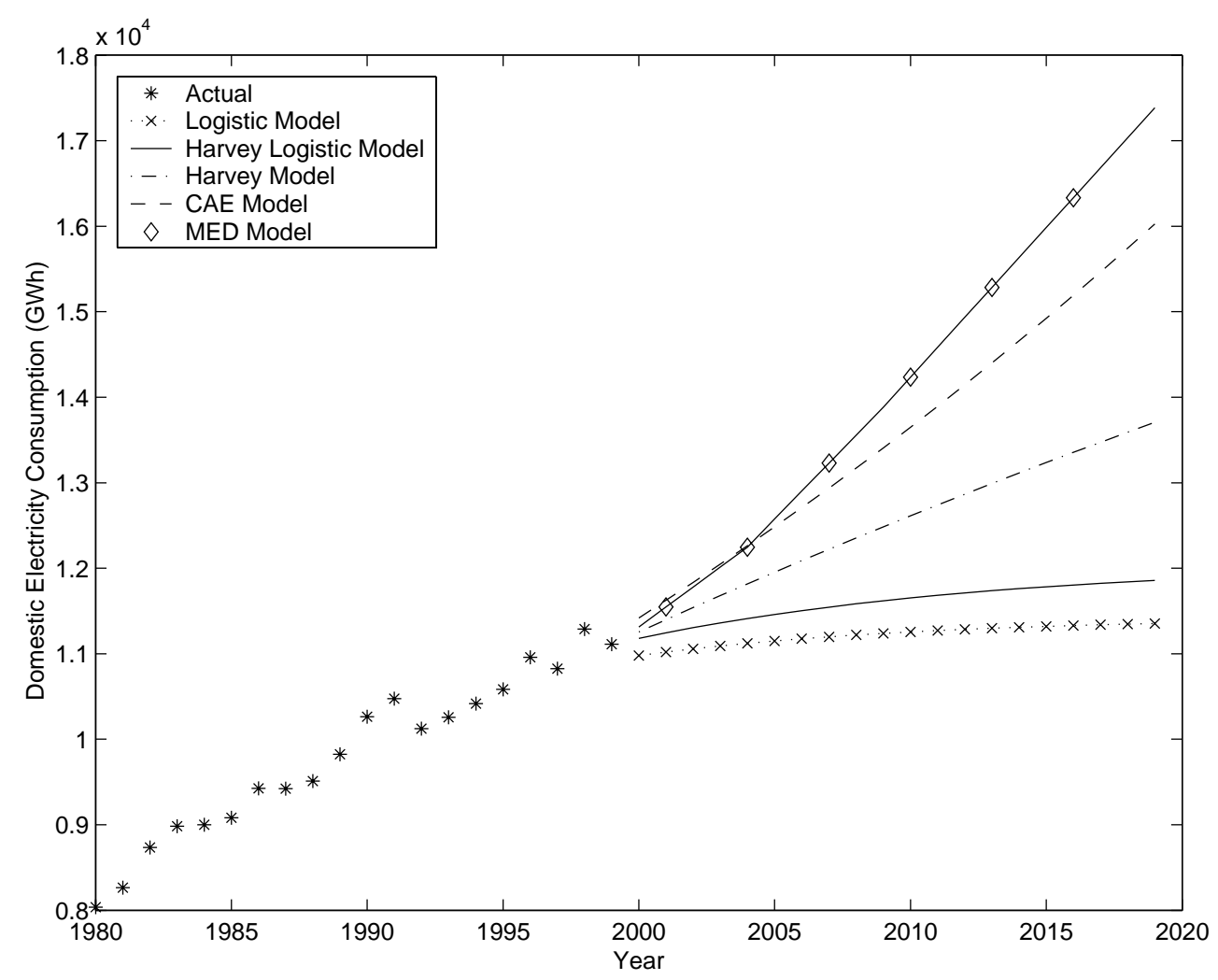

Fig. 8. Comparison of the models with the national forecasts for Domestic sector 


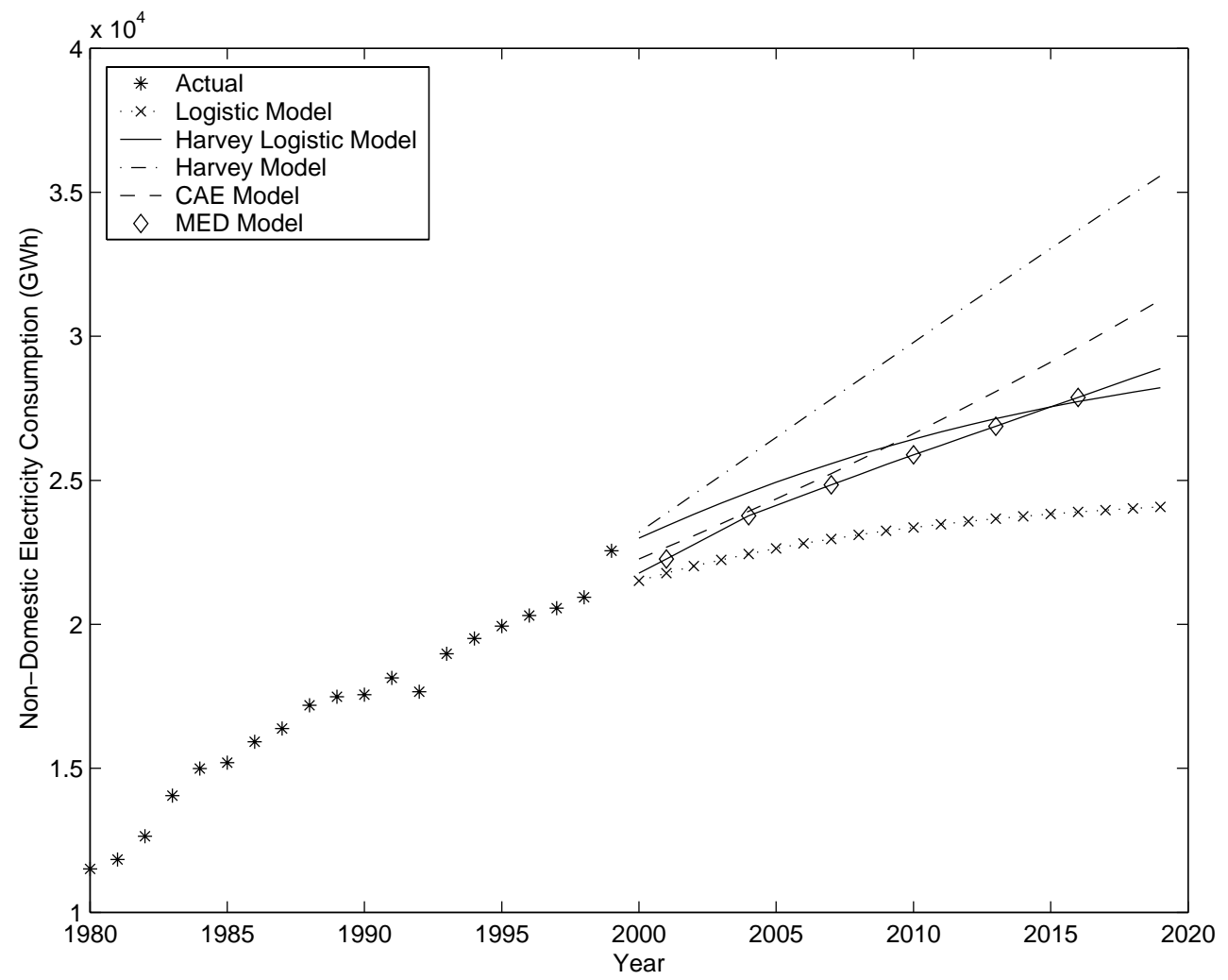

Fig. 9. Comparison of the models with the national forecasts for Non-Domestic sector 


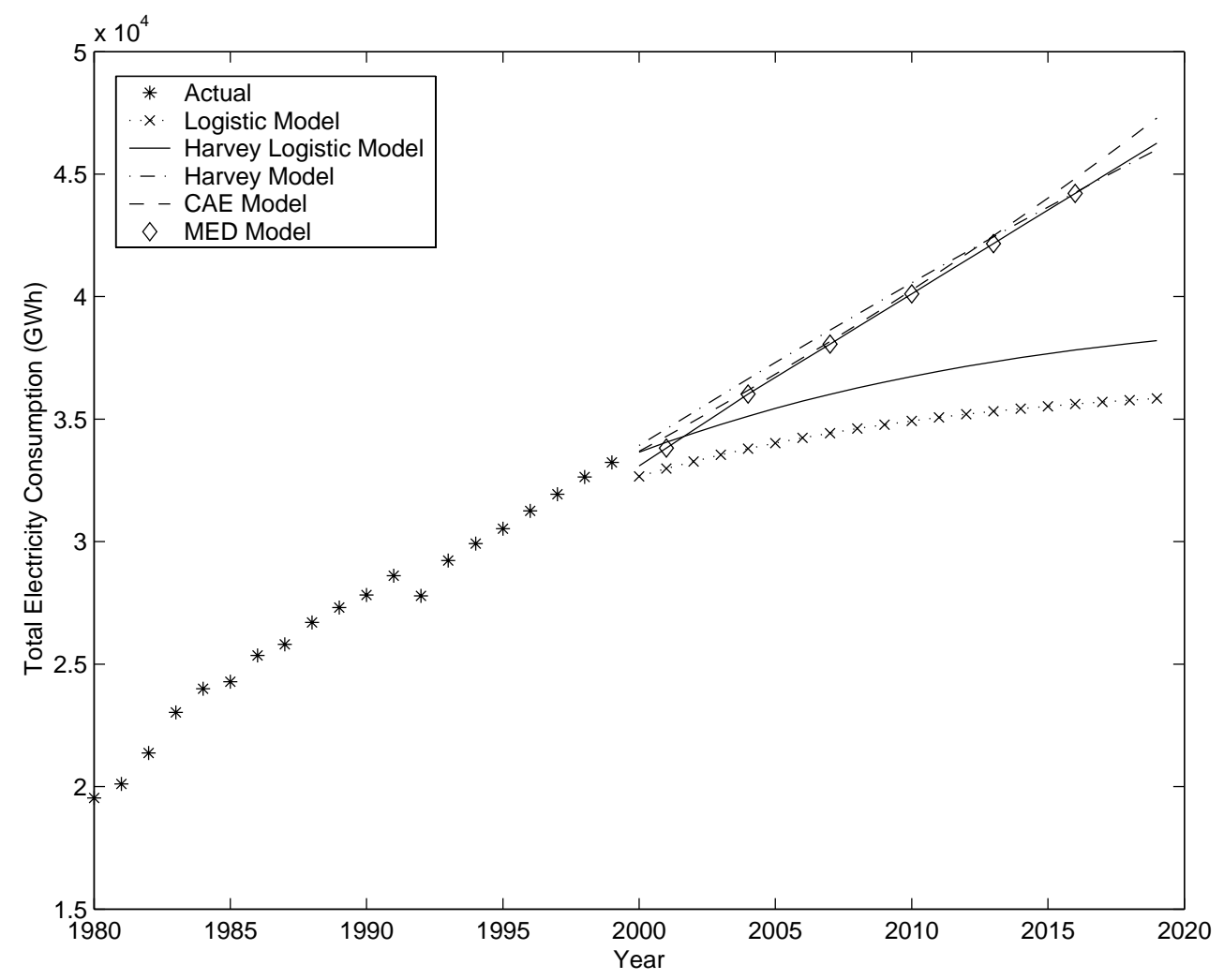

Fig. 10. Comparison of the models with the national forecasts for Total electricity consumption 